Vietnam Journal of Mechanics, NCST of Vietnam Vol. 25, 2003, No 2 (85-96)

\title{
APPLICATION OF NUMERICAL WATER FORCE TO A SMALL SPORT BOAT RUNNING ON A LAKE
}

\author{
H. T. Tran, S. Bikówski, C. D. Wolf, P. Maißer \\ Institute of Mechatronics at the Chemnitz University of Technology, \\ Reichenhainer Str. 88, D-09126 Chemnitz, Germany
}

\begin{abstract}
In this paper the motion of a small sport boat running on a lake is simulated using alaska tools. External forces and torques from water during movement have been taken into account. Using results from measurements the simulation has been validated.

Keywords. multibody system equations, fluid forces and torques to running boat, validations by measurement.
\end{abstract}

\section{Introduction}

The water force to a boat running on a sea or lake is investigated in recent years. Except the hydrostatic force and the flow resistance to the boat there are the water force and torque influence on the boat during running time. These force and torque are depending on added mass coefficients, acceleration and velocity components of the boat (see Newman 1985). Using the finite boundary element method we can calculate these added mass coefficients. Therefore, we will get the water force and torque acting on the boat during movement. The boat is considered as a multibody system. In this problem we have to simulate the motion of this Multibody System (see Maißer 1988, 1997) under the influence of the water external force and torque on the boat. To calculate this motion of the boat we use alaska tools.

\section{Formulation of the problem}

Let us consider a small boat running on a lake. This boat is regarded as a multibody system (MBS) and consists of two kayaks used as hull. Between thern, the articulated four bar driving system is placed. This mechanism is protected by patent which transforms the displacement of the driver standing on the lower platform into a vertical movement of the fin (figure 1).

To investigate the behavior of the boat, a multibody alaska model is used. The degree of freedom of the model and the number of bodies depend on the kind and number of fins. Considering only the boat driving system, there are 6 bodies in the MBS having a degree of freedom (DOF) of 10.

Regarding to the aim to build an optimal fin driven boat, two kinds of fins are available: an elastic fin and a rigid profile fin. The hull, the rear band, the lower platform and the front band compose the four bar mechanism representing a 
constrained multibody system (CMS). The driver modeled as a simple rigid body is connected by a rotational joint to the lower platform. By a constrained motion in this joint, an excitation can be applied to the MBS. The other person is fixed in the front of the boat as a passenger not moving.

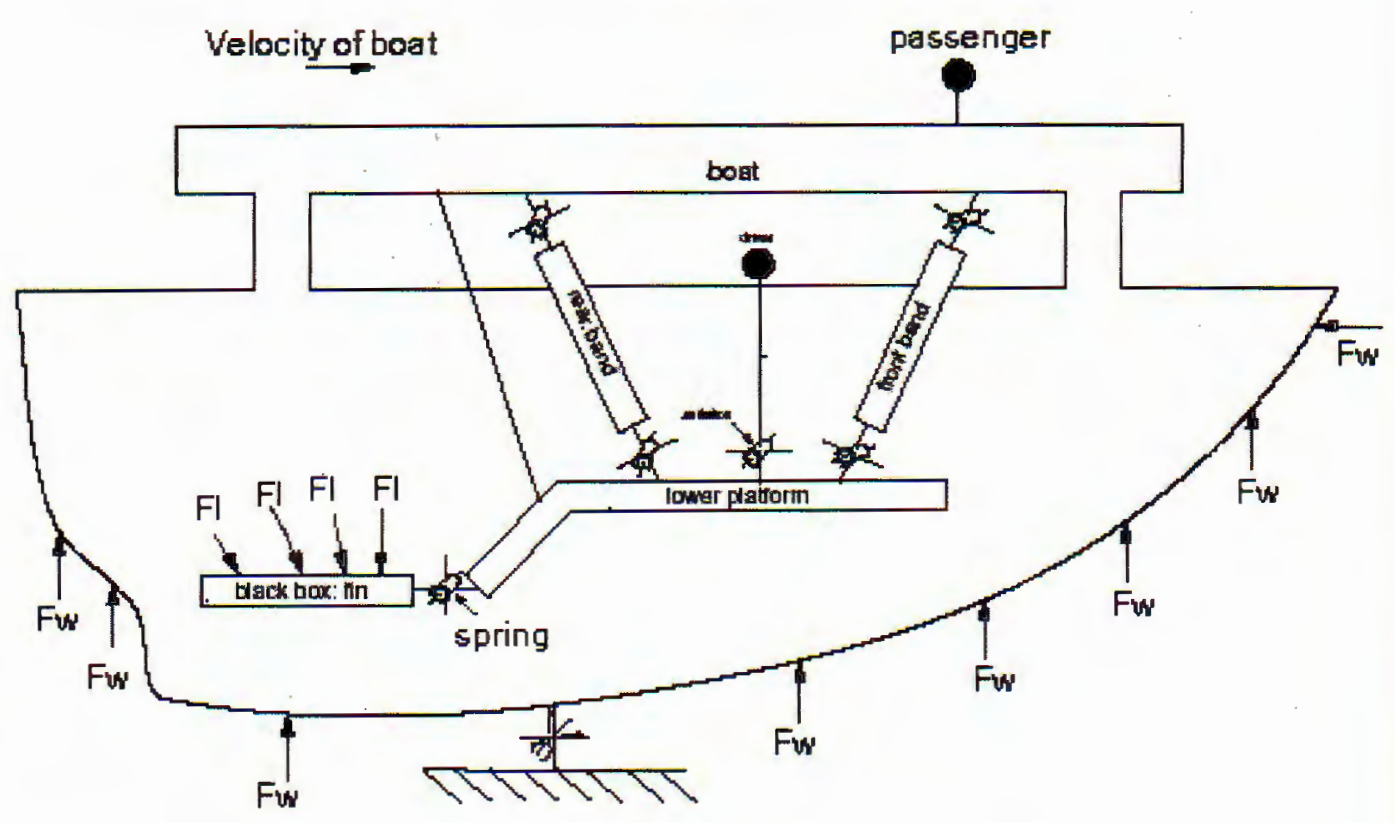

Fig. 1. Model of fin driven boat

As shown in Maißer 1988 and 1997 the motion equations of an MBS (Lagrange equation) are written in explicit form

$$
\begin{aligned}
& g_{a b}(q, t) \ddot{q}^{b}+\Gamma_{a b c}(q) \dot{q}^{b} \dot{q}^{c}=Q_{a} \\
& g_{a b}=\sum_{k=1}^{K}\left(m_{k} \partial_{a}{\underset{k}{X}}^{(i)} \partial_{b} \underset{k}{X^{(i)}}+\underset{k}{\vartheta^{i j}} \partial_{a} \underset{k}{E_{i}^{(i)}} \partial_{b} E_{k}^{(i)}\right)=g_{b a}
\end{aligned}
$$

denotes the $V^{n}$-metric,

$$
\begin{aligned}
\Gamma_{a b c} & :=\frac{1}{2}\left(\partial_{b} g_{a b}+\partial_{c} g_{a b}-\partial_{a} g_{b c}\right) \\
& =\sum_{k=1}^{K}(m_{k} \partial_{a} \underset{k}{X^{(i)}} \partial_{b} \partial_{c} \underset{k}{X^{(i)}}+\underset{k}{\vartheta^{i j}} \partial_{a} \underset{k}{E_{i}^{(i)}} \partial_{b} \partial_{c} \underbrace{E_{j}^{(i)}}_{k})=\Gamma_{a c b}
\end{aligned}
$$

are the Christoffel symbols of the first kind and

$$
Q_{a}=\sum_{k=1}^{K}\left(\underset{k}{K^{(i)}} \partial_{a} \underset{k}{X^{(i)}}+\frac{1}{2} \underset{k}{M^{(i j)}} \partial_{a} \underset{k}{E_{i}^{(i)}} \underset{k}{E_{j}^{(i)}}\right)
$$


denote the generalized forces, where $\underset{k}{X^{(i)}}$ are the Cartesian coordinates of the center of mass of the body $B_{k}, K_{k}^{(i)}$ are the components of all applied forces acting on the body $B_{k}, M_{k}^{(i j)}$ is the two-dimensional skew-symmetric tensor of the resultant of all applied torques acting on the body $B_{k}, m_{k}$ denote the mass of the body $B_{k}$ and $\vartheta_{k}^{i j}$ its BINET's inertia tensor, $k=1, \ldots, K$ and $K$ is the number of bodies of an MBS. Indices in brackets refer to the inertial frame, without brackets to the body frame.

\section{The water forces acting on the boat running in a lake}

In this part we will see the force and torque of fluid acting on the moving boat. The fluid is assumed to be ideal and irrotational.

With respect to formula (2.4) of generalized forces there are the force and torque from water during the running time of the boat. These water force and torque acting on the boat during movement are written (Newman 1985):

$$
\begin{aligned}
\mathfrak{F} & =-\rho \frac{\mathrm{d}}{\mathrm{dt}} \iint_{S_{B}} \phi \mathfrak{n d} S_{B}, \\
\mathfrak{M} & =-\rho \frac{\mathrm{d}}{\mathrm{dt}} \iint_{S_{B}} \phi(\mathfrak{R} \times \mathfrak{n}) \mathrm{d} S_{B} .
\end{aligned}
$$

Here, $\phi$ denotes the velocity potential of water, $\mathfrak{R}=\mathfrak{R}_{0}(t)+\mathfrak{r}$ is the vector from the origin of the inclined frame to body fixed origin $(\mathfrak{r}=0)$, the normal vector $\mathfrak{n}$ is taken to be positive oriented and pointing out of the water volume.

If the translation velocity is $\mathfrak{V}(t)$ and the body is rotating with the angular velocity $\vec{\omega}(t)$, the velocity potential must satisfy the boundary condition on the body surface $S_{B}$

$$
\frac{\partial \phi}{\partial n}=\mathfrak{V} \cdot \mathfrak{n}+\vec{\omega} \cdot(\mathfrak{r} \times \mathfrak{n}) .
$$

Here $\mathfrak{V}=\left(V_{1}, V_{2}, V_{3}\right), \vec{\omega}=\left(\omega_{1}, \omega_{2}, \omega_{3}\right) \equiv\left(V_{4}, V_{5}, V_{6}\right)$.

Thus $V_{1}, V_{2}, V_{3}$ denote the three components of translation velocity (surge, heave and sway) and $\omega_{1}=V_{4}, \omega_{2}=V_{5}, \omega_{3}=V_{6}$ denote the corresponding rotational velocity components (roll, yaw, pitch). The boundary condition (3.3) suggests that the total potential can be expressed by the sum $\phi=V^{j} \phi_{j}(j=1, \ldots, 6)$, where each component $\phi_{j}$ satisfies the corresponding condition:

$$
\begin{aligned}
& \frac{\partial \phi_{j}}{\partial \mathfrak{n}}=\mathfrak{n}_{j}, \quad j=1,2,3, \\
& \frac{\partial \phi_{j}}{\partial \mathfrak{n}}=(\mathfrak{r} \times \mathfrak{n})_{j-3}, \quad j=4,5,6 .
\end{aligned}
$$


Therefore, if the boat is moving in the water with the velocity $U$ in the $x$-direction with respect to inertial frame, then the potential velocity will satisfy the following system of equations (see Timman, Hermans and Hsiao 1985 and Wehausen and Laiton 1960):

$$
\begin{aligned}
\Delta \phi_{j} & =0 \text { except at }(\xi, \eta, \zeta), \\
\frac{\partial^{2} \phi_{j}}{\partial x^{2}}+\frac{g}{U^{2}} \frac{\partial \phi_{j}}{\partial y} & =0 \text { on the free surface } y=0, j=1, \ldots, 6, \\
\frac{\partial \phi_{j}}{\partial y} & =0 \text { on the bottom } y=-h, \\
\lim _{x \rightarrow \infty} \operatorname{grad} \phi_{j} & =0, \\
\frac{\partial \phi_{j}}{\partial \mathfrak{n}} & =\mathfrak{n}_{j}, j=1,2,3 \text { on } S_{B}, \\
\frac{\partial \phi_{j}}{\partial \mathfrak{n}} & =(\mathfrak{r} \times \mathfrak{n})_{j-3}, \quad j=4,5 ; 6 \text { on } S_{B} .
\end{aligned}
$$

The Green's formula of this problem is (Wehausen and Laiton 1960):

$$
\begin{aligned}
& G(x, y, z, \xi, \eta, \zeta)=\frac{1}{r_{1}}+\frac{1}{r_{2}}-\frac{1}{4} \int_{0}^{\pi / 2} \mathrm{~d} \tau \mathrm{PV} \int_{0}^{\infty} \frac{e^{-k h} \cosh k(y+h)}{k \cos ^{2} \tau \cosh k h-\nu \sinh k h} \times \\
& {\left[\cosh k(\eta+h)\left(k \cos ^{2} \tau+\nu\right)-\nu\right] \times \cos [k(x-\xi) \cos \tau] \cos [k(z-\zeta) \sin \tau] \mathrm{d} k} \\
& -4 \int_{\tau_{0}}^{\pi / 2} \frac{e^{-k_{0} h} \operatorname{sech} k_{0} h \cosh k_{0}(y+h)}{\cos ^{2} \tau-\nu h \operatorname{sech}^{2} k_{0} h} \times\left[\cosh k_{0}(\eta+h)\left(k_{0} \cos ^{2} \tau+\nu\right)-\nu\right] \times \\
& \sin \left[k_{0}(x-\xi) \cos \tau\right] \cos \left[k_{0}(z-\zeta) \sin \tau\right] \mathrm{d} \tau
\end{aligned}
$$

with $\nu=\frac{g}{U^{2}}, \quad r_{1}=\sqrt{(x-\xi)^{2}+(y-\eta)^{2}+(z-\zeta)^{2}}$,

$r_{2}=\sqrt{(x-\xi)^{2}+(y+2 h+\eta)^{2}+(z-\zeta)^{2}} . \quad k_{0}=k_{0}(\tau)$ denotes the real positive root of the following equation:

$k_{0}-\nu \sec ^{2} \tau \tanh k_{0} h=0, \tau_{0}<\tau<\pi / 2$,

$\tau_{0}=\arccos \sqrt{\nu h}$ if $\nu h<1, \tau_{0}=0$ if $\nu h \geq 1$. Using the result of Wehausen and Laiton 1960, we can find the potential velocity function $\phi_{j}(j=1, \ldots, 6)$ by the following formula:

$$
\phi_{j}(x, y, z)=\iint_{S_{B}} \gamma(\xi, \eta, \zeta) G(x, y, z, \xi, \eta, \zeta) \mathrm{d} S_{B}
$$

$\gamma(x, y, z)$ denotes the solution of the following equation:

$$
\phi_{j n}(x, y, z)=2 \pi \gamma(x, y, z)+\iint_{S_{B}} \gamma(\xi, \eta, \zeta) G_{n}(x, y, z, \xi, \eta, \zeta) \mathrm{d} S_{B}
$$


here $(x, y, z) \in S_{B}$. Substituting the boundary conditions of the potential velocity $\phi_{j}$ on surface $S_{B}$ into the equation (3.6) we have

$$
n_{j}(x, y, z)=2 \pi \gamma(x, y, z)+\iint_{S_{B}} \gamma(\xi, \eta, \zeta) G_{n}(x, y, z, \xi, \eta, \zeta) \mathrm{d} S_{B}
$$

Here $n_{j}(x, y, z)(j=1,2,3)$ are the components of normal vector $\mathfrak{n}$ oriented into the body at the point $(x, y, z) \in S_{B}$ and $n_{4}=y n_{3}-z n_{2}, n_{5}=z n_{1}-x n_{3}, n_{6}=x n_{2}-y n_{1}$. For the resolution, the body surface $S_{B}$ is divided into $N$ panels as follows: $S_{B}=$ $\bigcup_{i=1}^{N} \triangle S_{n}$. By writing equation (3.7) at each center of panels we obtain $N$ equations for $N$ unknowns:

$$
h_{j m}=2 \pi \gamma_{j m}+\sum_{n=1}^{N} \gamma_{j n} \iint_{\Delta S_{n}} G_{n}\left(x_{m}, y_{m}, z_{m}, \xi_{n}, \eta_{n}, \zeta_{n}\right) \mathrm{d} S_{n},
$$

where $h_{j m}=n_{j}$ and $m=1, \ldots, N, j=1, \ldots, 6$.

Therefore, we can rewrite the system of equation (3.8) as follows:

$$
A \gamma_{j}=h_{j} \quad(j=1, \ldots, 6),
$$

here $A$ generally is a dense and nonsymmetrical matrix of $N$-order and $\gamma_{j}=$ $\left(\gamma_{j 1}, \ldots, \gamma_{j N}\right)$.

Substituting the vector solution $\gamma_{j}$ of equation (3.9) into the equation (3.6) we obtain the potential velocity $\phi_{j m}$ at the center point of the panel $\triangle S_{m} \in S_{B}(m=$ $1, \ldots, N, j=1, \ldots, 6)$ as follows:

$$
\phi_{j m}=\sum_{n=1}^{N} \gamma_{j n} \iint_{\Delta S_{n}} G\left(x_{m}, y_{m}, z_{m}, \xi_{n}, \eta_{n}, \zeta_{n}\right) \mathrm{d} S_{n} .
$$

The added mass coefficients are written as follows (see Newman 1985):

$$
m_{i j}=\rho \iint_{S_{B}} \phi_{i} \frac{\partial \phi_{j}}{\partial \mathfrak{n}} \mathrm{d} S_{B}, \quad i, j=1, \ldots, 6
$$

By a similar way of Timman, Hermans and Hsiao 1985, we can prove $m_{i j}=m_{j i}$. Indeed, using Green's formula for potential functions $\phi_{j}$ and $\phi_{i}(i, j=1, \ldots, 6)$ we have:

$$
\iint_{S_{B}}\left(\phi_{i} \frac{\partial \phi_{j}}{\partial \mathfrak{n}}-\phi_{j} \frac{\partial \phi_{i}}{\partial \mathfrak{n}}\right) \mathrm{d} S_{B}=-\frac{g}{U^{2}} \iint_{S_{0}}\left(\phi_{i} \frac{\partial^{2} \phi_{j}}{\partial x^{2}}-\phi_{j} \frac{\partial^{2} \phi_{i}}{\partial x^{2}}\right) \mathrm{d} S_{0}
$$


here $S_{0}$ is the free surface of the fluid $(y=0)$. From this formula we can carry out the integration with respect to $x$ then the above expression can be rewritten in the form:

$$
-\frac{U^{2}}{g} \int\left(\phi_{i} \frac{\partial \phi_{j}}{\partial x}-\phi_{j} \frac{\partial \phi_{i}}{\partial x}\right)_{C} \mathrm{~d} z
$$

Here $C$ stands for the corresponding $x$ boundary of $S_{0}$ wave. At infinity this integrated terms vanish and then we have

$$
\rho \iint_{S_{B}} \phi_{i} \frac{\partial \phi_{j}}{\partial \mathfrak{n}} \mathrm{d} S_{B}=\rho \iint_{S_{B}} \phi_{j} \frac{\partial \phi_{i}}{\partial \mathfrak{n}} \mathrm{d} S_{B} \quad \text { or } \quad m_{i j}=m_{j i} .
$$

Using the formula (3.10) and the boundary conditions of the potential velocity $\phi_{j}$ on the surface $S_{B}$ the coefficients $m_{i j}$ can be calculated by the following formula:

$$
\begin{aligned}
m_{i j} & =\iint_{S_{B}}\left(\sum_{n=1}^{N} \gamma_{i n} \iint_{\Delta S_{n}} G\left(x_{m}, y_{m}, z_{m}, \xi_{n}, \eta_{n}, \zeta_{n}\right) \mathrm{d} S_{n}\right) \mathfrak{n}_{j} \mathrm{~d} S_{B} \\
& =\sum_{m=1}^{N}\left(\sum_{n=1}^{N} \gamma_{i n} \iint_{\Delta S_{n}} G\left(x_{m}, y_{m}, z_{m}, \xi_{n}, \eta_{n}, \zeta_{n}\right) \mathrm{d} S_{n}\right) h_{j m} \Delta S_{m} .
\end{aligned}
$$

Here $i, j=1, \ldots, 6$ and $\triangle S_{k} \in S_{B}(k=1, \ldots, N)$.

Using the result of Newman 1985 , we have the formulas of the forces and torques with respect to the $k$-body fixed reference frame (BFR):

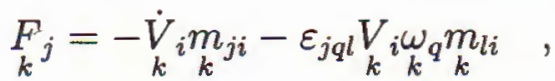

$$
\begin{aligned}
& \underset{k}{M_{j}}=-\dot{V}_{k} \underset{k}{m_{j+3, i}}-\varepsilon_{j q l} V_{k}{\underset{k}{\omega}}_{k} m_{k} m_{l+3, i}-\varepsilon_{j q l} V_{k} V_{k} V_{k} m_{k i} .
\end{aligned}
$$

Here $j, q, l$ take the values $1,2,3$ and as the index $i$ is used to denote the six components of the velocity potential $i=1, \ldots, 6, \varepsilon_{j q l}$ denotes the LEVI-CIVITA symbol. In (3.4) the added mass coefficients are depending on the geometry of body and on the velocity $U$. From formula (3.12) it is clear that the water force and moment are depending on acceleration $\dot{V}_{k}(t)$ and velocity ${ }_{k}(t)$ of $k$-body. Substituting the force and torque (3.12) into formula (2.4) we have the generalized force from the water to the boat. But if we put all this exterior generalized force to the right side of Langrange's equation (2.1) then our program is shut-down because the acceleration of the boat at the first moment is too big so the following force also is too large. So in the calculation we put the force terms depending on acceleration to the left side of Langrange's equations then we have the generalized Lagrange's equations with modified metric and generalized Christoffel's symbol. Therefore, our 
calculation approach is stable. Denote the force terms depending on the acceleration components by virtual mass and the terms depending on velocity components by virtual damping. In some figures we will take the interval of time only from 40 seconds to 50 seconds because after about 30 seconds our calculation comes to stationary state. We have calculated the boat motions with and without water force and torque during movement. Taking into account these water force and torque the movement range of center of mass of the boat by vertical direction is smaller. That means, in this case of simulation the boat is running more quietly. In figure 2 we can see the difference between two movement ranges of center of mass of the boat with and without applying these force and torque. Figure 3 and figure 4 show the force and torque to the boat during movement. Figure 5, figure 6 and figure 7 , figure 8 show the force and torque parts depending on acceleration and velocity of the boat. Figure 3 and figure 5 look similar because in the first equation of formula (3.12) the force term depending on acceleration is very big in comparing with the other force term. In those figures the forces by $z$ direction and the torques about $y$ direction are zero because our model is a two-dimensional one.

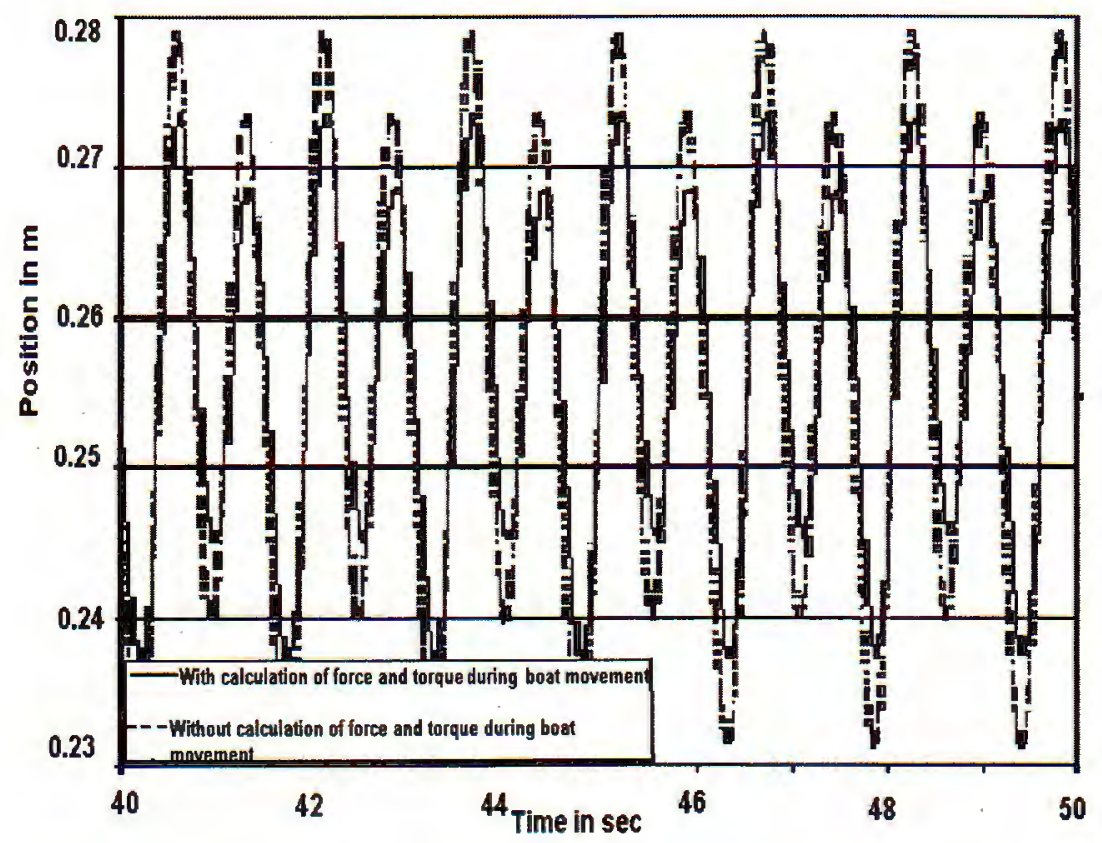

Fig. 2. Position of center of mass of the boat by vertical direction 


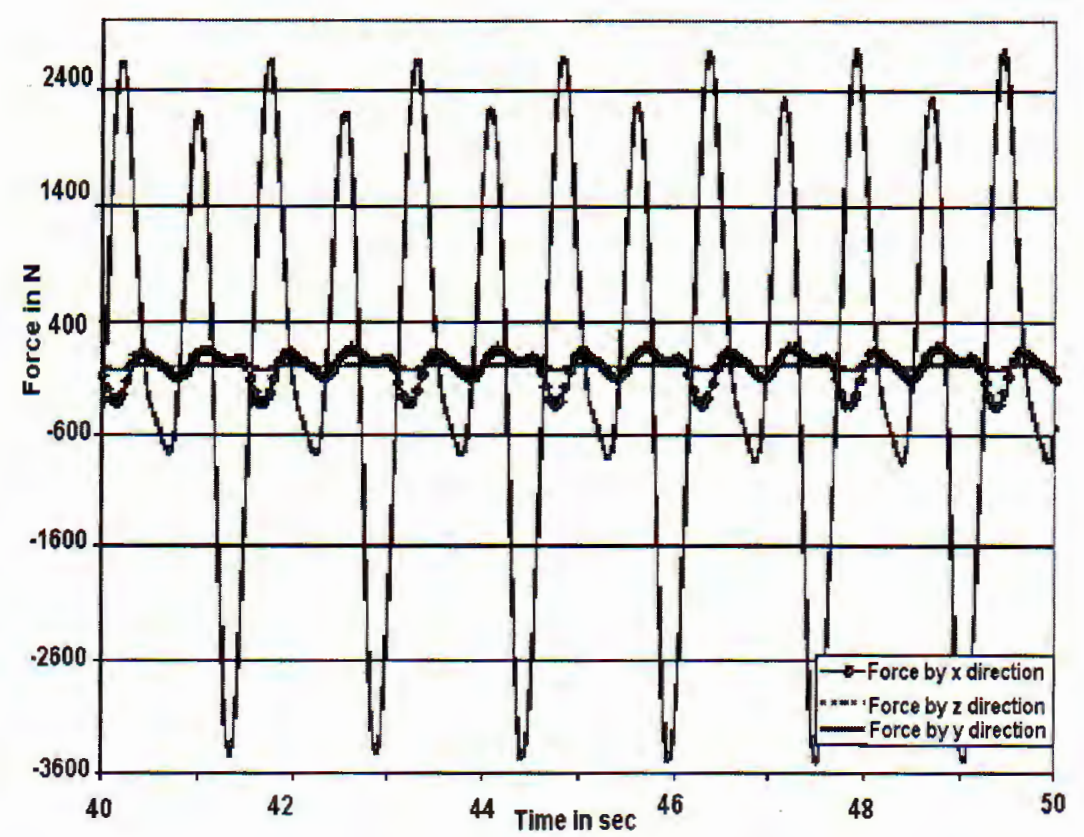

Fig. 3. Force by $x, z, y$ directions to the boat during movement

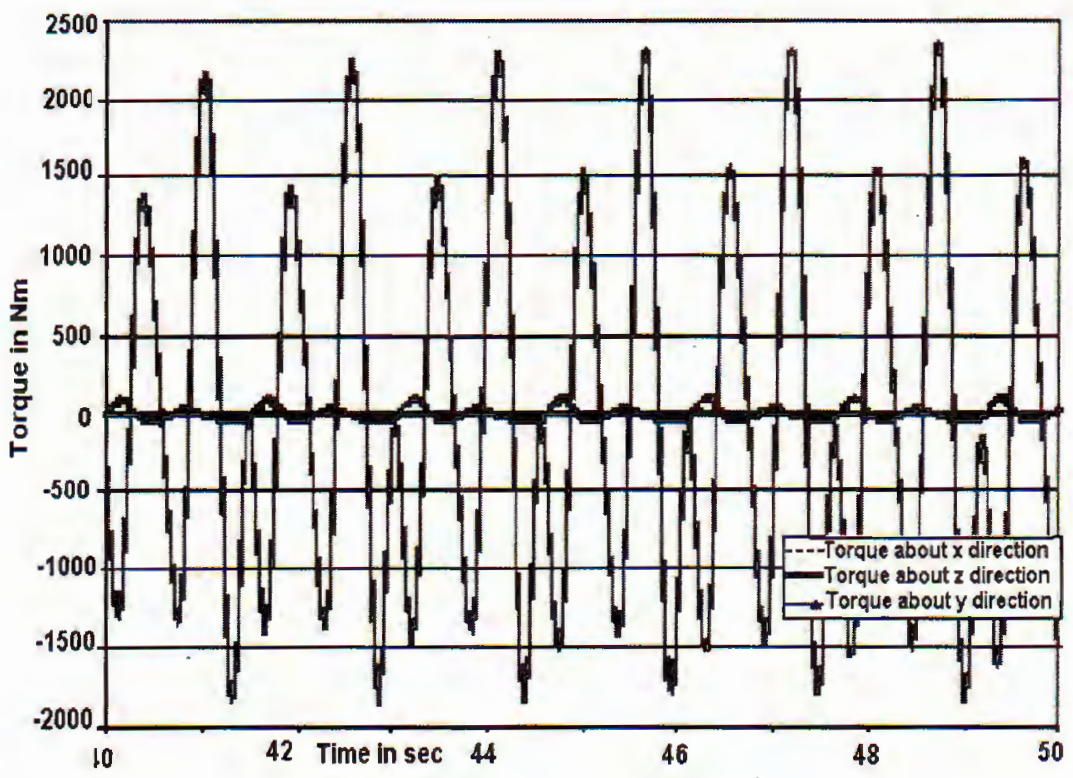

Fig. 4. Torque about $x, z, y$ directions to the boat during movement 


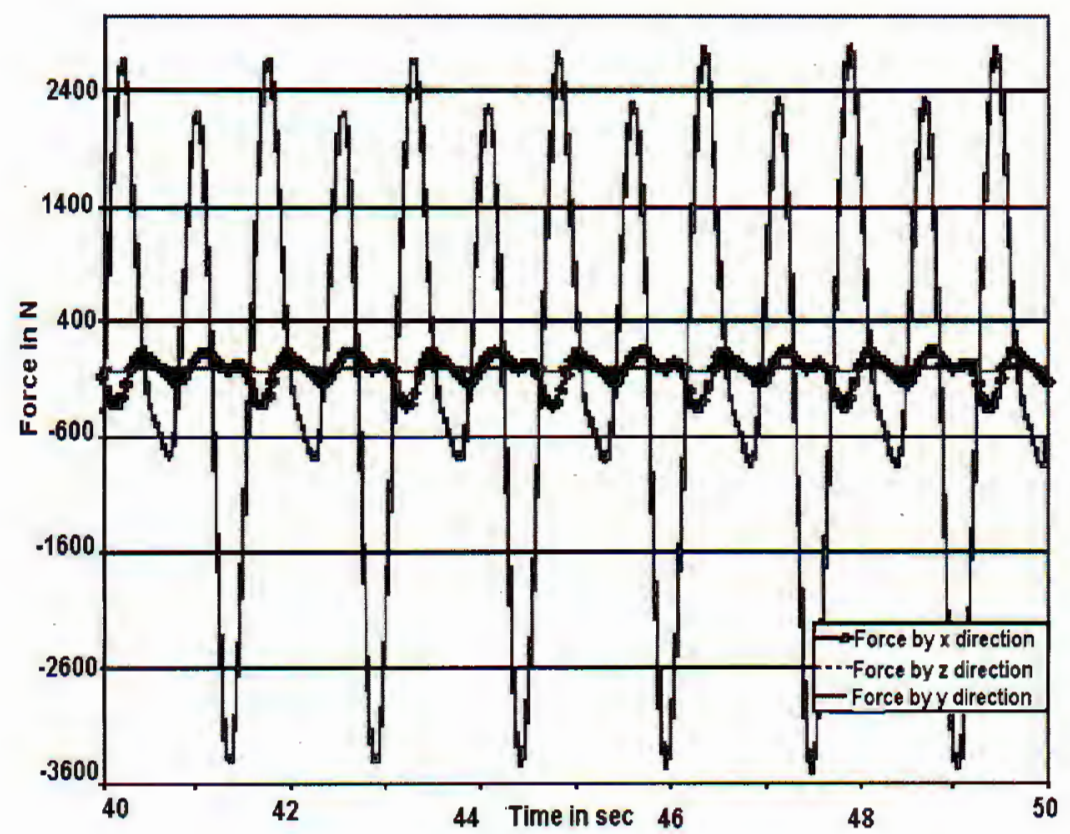

Fig. 5. Force by $x, z, y$ directions to the boat depending on acceleration components

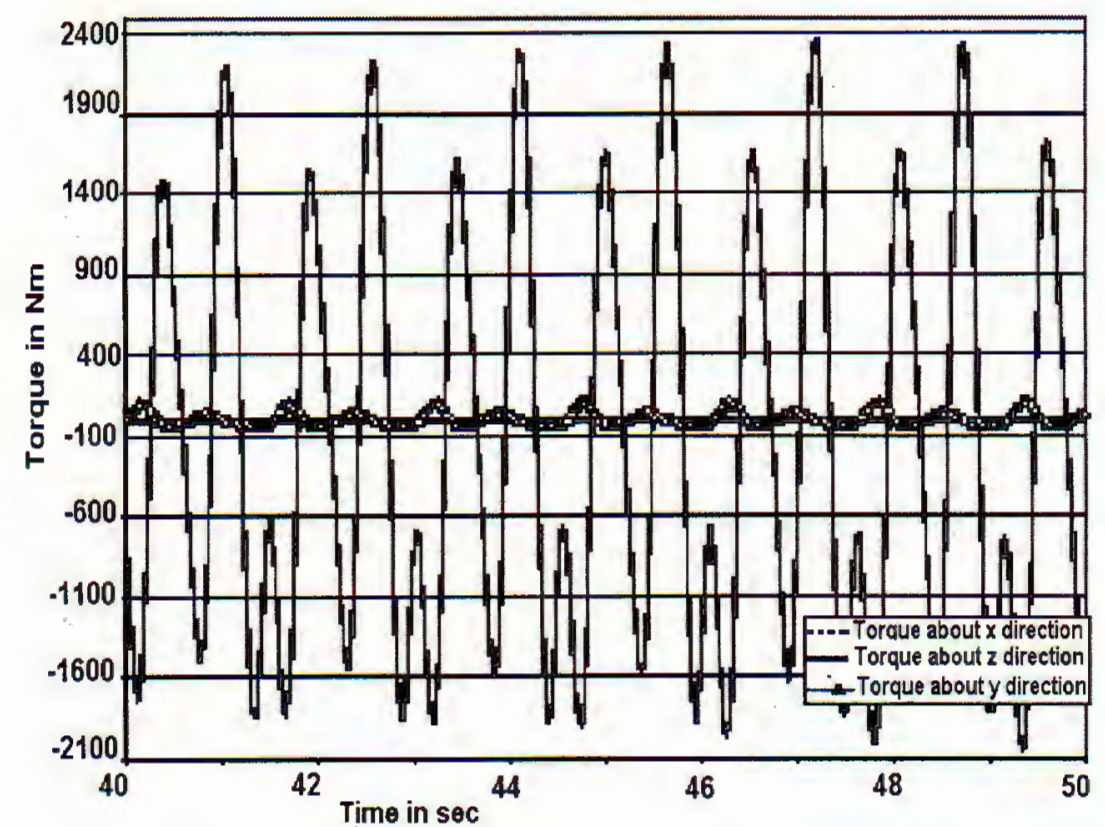

Fig. 6. Torque about $x, z, y$ directions to the boat depending on acceleration components 


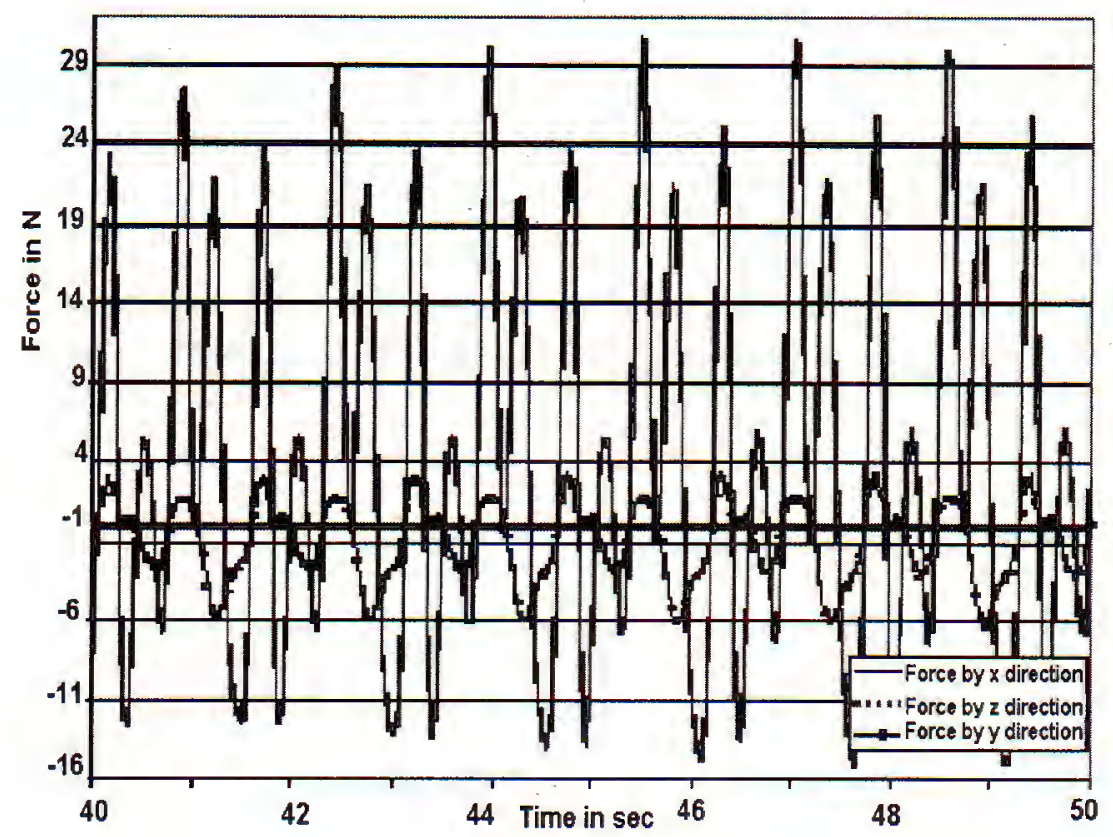

Fig. 7. Force by $x, z, y$ directions to the boat depending on velocity components

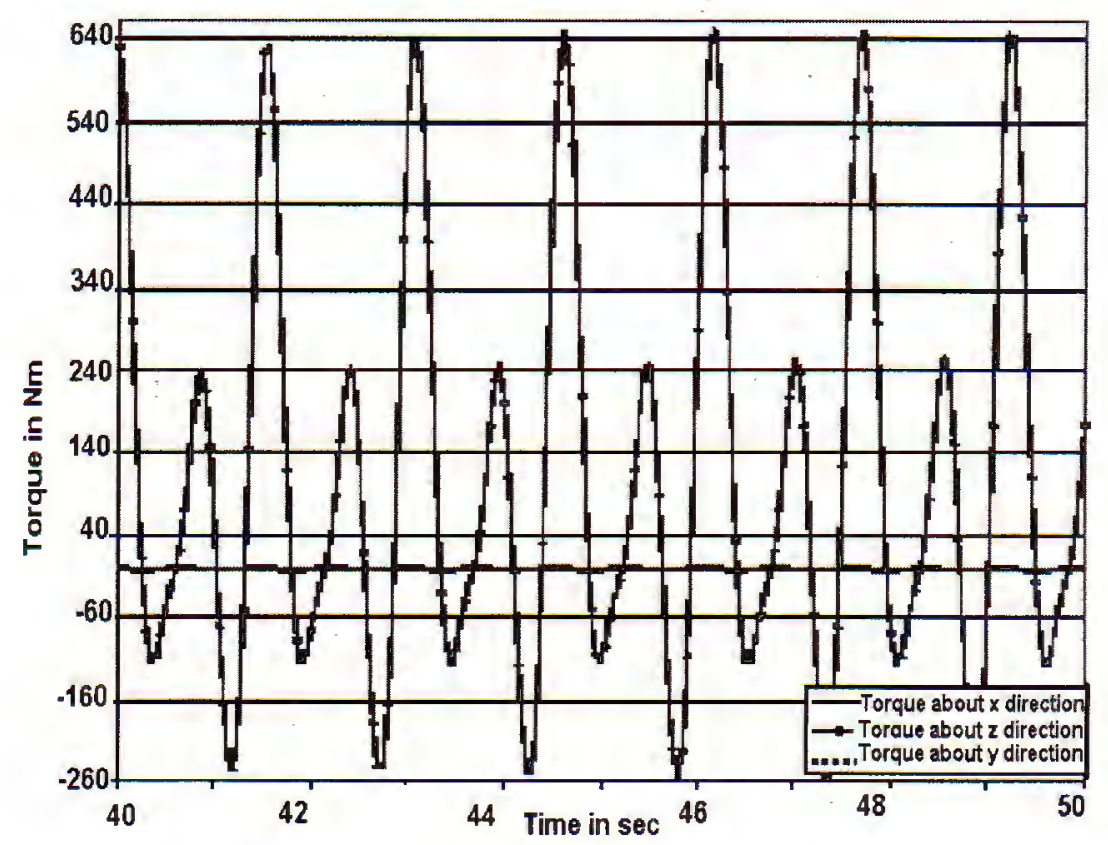

Fig. 8. Torque about $x, z, y$ directions to the boat depending on velocity components 


\section{Result in validation of the velocities of the boat}

By experiments we have done several measurements to get the velocities of the boat with different models of fins under some excitation frequencies of the person. In this part we introduce one of our results in comparison with measurement. Figure 9 shows the velocity of the boat with one profile fin in the front. From this figure we can see that after 50 seconds the behavior and the mean values of velocities in calculations and measurements are similar.

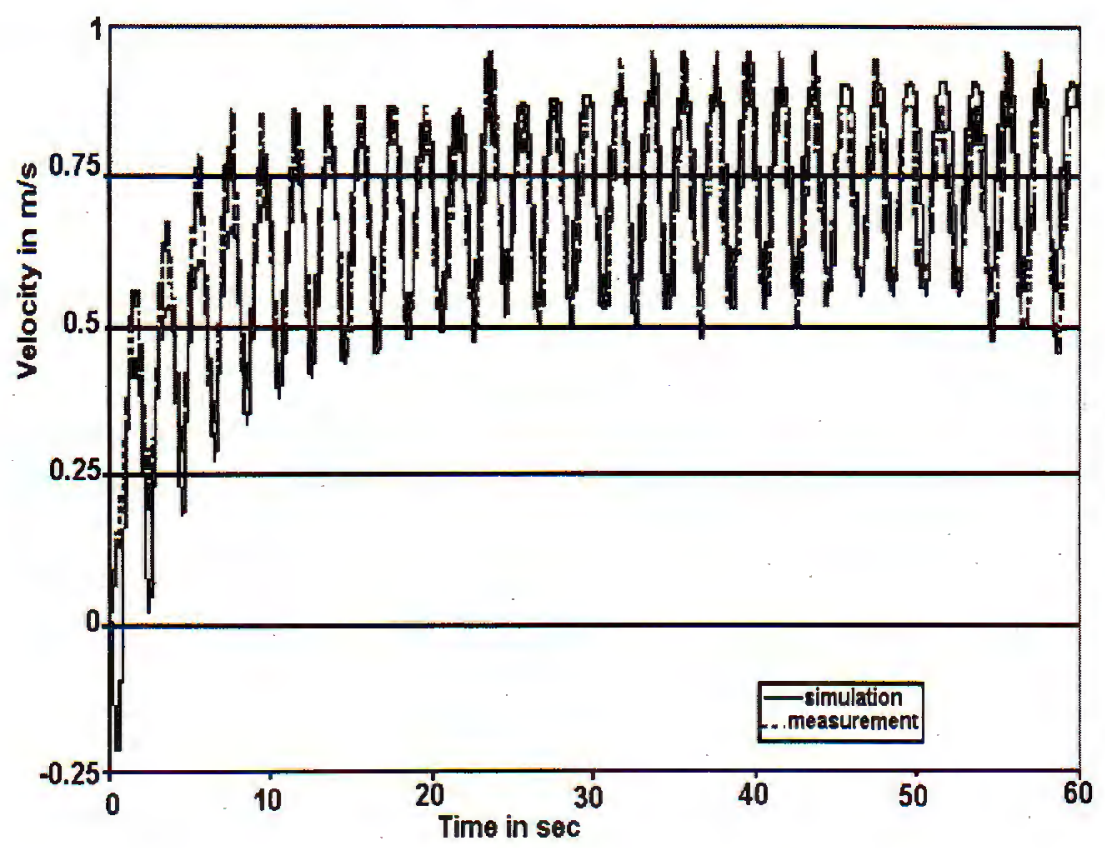

Fig. 9. Boat velocity-one elastic fin in front

\section{Conclusion.}

The problem to describe the motions of the fin driven boat running on a lake is difficult because of many influences acting on the boat. In this paper we have presented only the water force and torque to the boat during running time. Using the model of boat with all external forces and torques from water and excitation of the person we can do an optimization of kinetic variations and get a good design of the boat.

\section{REFERENCES}

1. Maißer P. Differential geometric methods in Multibody dynamics. Nonlinear Analysis, Theory, Methods and Applications, 30(8) (1997), 5127-5133. 
2. Maißer P. Analytische Dynamik von Mehrkörpersystemen. ZAMM, 68(10) (1988), 463-481.

3. Byron Bird R., Robert C. Armstrong, Ole Hassager. Dynamics of polymeric liquids - Volume 1 Fluid Mechanics. A Wiley-Interscience Publication, 649, 1987.

4. Stoker J. J. Water waves - The mathematical theory with application. A Wiley - Interscience Publication, 567, 1992.

5. Timman R., Hermans A. J., Hsiao G. C. Water waves and ship hydrodynamics. Martinus Nijhoff Publishers, 145, 1985.

6. Newman J. N. Marine Hydrodynamics. The MIT Press Cambridge, Massachussetts and London England, 395, 1985.

7. Wehausen J. V. and Laiton E. V. Surface waves. In: Encyclopedia of Physics, Berlin: Springer 9:446-778, 1960.

8. Chakrabarti S. K. Hydrodynamics of offshore structures. Computational Mechanics Publications Southampton Boston, Springer-Verlag, 440, 1987.

Received February 7, 2002

\section{ÁP DỰG TÍNH TOÁN LỰC NƯớC \\ LÊN TÀU THỂ THAO NHỎ CHAYY TRÊN HỒ}

Trong bài báo này sự chuyển động của tàu thể thaọ nhỏ chạy trên hồ được mô phỏng bằng cách sừ dụng công cụ Alaska. Lực và mô men ngoài từ nước trong suốt thời gian chuyển động được tính đến. Các kết quả tính được so sánh với các kết quả đo. 